\title{
A Study on Gastro Instestinal Stromal Tumors: A Risk of Metastasis
}

\author{
Dr. Siva Ranjan $D^{1}$, Dr. Majety Dora Venkata Ramnath ${ }^{2 *}$
}

${ }^{1}$ Associate Professor, ${ }^{2}$ Assistant Professor, Nimra Institute of Medical Sciences, 4-90, Nimra Nagar, Ibrahimpatnam, Jupudi, Vijayawada, Andhra Pradesh 521456, India

DOI: $10.36348 /$ sjpm.2020.v05i06.003 $\quad$ | Received: 09.06.2020 | Accepted: 17.06 .2020 | Published: 21.06 .2020

*Corresponding author: Dr. Majety Dora Venkata Ramnath

\section{Abstract}

Background: Stromal tumors arising in GastroIntestinal tract were generally regarded as smooth muscle neoplasms such as leiomyoma, leiomyosarcoma, leiomyoblastoma and bizarre leiomyomas but relatively few of these neoplasms had convincing ultrastructural evidence of smooth muscle differentiation. Aims \& objectives: The main aim of the study is to review cases of Gastrointestinal Stromal Tumors and to categorize these neoplasms based on the "risk of metastasis". Materials \& methods: The study was done for three years and we received 30 specimens consisted of resections and biopsies from gastrointestinal tract in the department of pathology and diagnosed as Gastrointestinal Stromal Tumors, for which we assessed the risk of metastasis. Results: There was a preponderance of high risk Gastrointestinal Stromal Tumors with 21 cases (70\%), followed by intermediate risk 5 cases (16.67\%), low risk 2 cases $(6.67 \%)$ and very low risk 2 cases $(6.67 \%)$. Conclusion: Gastrointestinal stromal tumors are a heterogenous group of neoplasms. With the advent of targeted therapy using gleevac the responsibility of the pathologists has increased to give a specific diagnosis. It is also important to suggest the prognosis based on the pathological findings.

Keywords: Gastrointestinal Stromal Tumors, Metastasis, Spindle cell neoplasms, Histopathology.

Copyright @ 2020: This is an open-access article distributed under the terms of the Creative Commons Attribution license which permits unrestricted use, distribution, and reproduction in any medium for non-commercial use (NonCommercial, or CC-BY-NC) provided the original author and sources are credited.

\section{INTRODUCTION}

In 1940s by Stout and others, stromal tumors arising in Gastrointestinal tract were generally regarded as smooth muscle neoplasms (leiomyoma, leiomyosarcoma, leiomyoblastoma and bizarre leiomyomas) but relatively few of these neoplasms had convincing ultrastructural evidence of smooth muscle differentiation [1].

Use of immunohistochemistry (IHC) in early 1980s showed that many of these lesions lacked evidence of smooth muscle differentiation which led few authors in 1983 to introduce generic designation "stromal tumours" [2]. It was observed that some stromal tumours of GI tract expressed neural crest antigens like S-100 protein and neuron specific enolase. Electronmicroscopically some cases showed schwannian or neuro axonal differentiation. In a study done in 1984 in a subset of such lesions showed clear ultrastructural evidence of autonomic neuronal differentiation and described them as plexosarcoma. These subsequently became better known as gastrointestinal autonomic nerve tumors [3].
The diagnostic reproducibility was flayed because of varying levels of expertise and lack of specific diagnostic marker that could reliably identify non myogenic and non schwannian stromal tumours. The revelation concerning KIT mutations and KIT expressions have rapidly transformed the field. The KIT immunoreactivity in specific context of mesenchymal lesions of GI tract defines a group of tumours showing differentiation towards (or derived from) interstitial cells of Cajal. True smooth muscle neoplasms and schwann cell neoplasms of GI tract lack KIT mutations and donot express CD-117 [4]. Whether or not KIT positivity should be required for diagnosis of GISTs is more contentious. The clinicopathological features of GIST are so characteristic that some experts may feel secure in making the diagnosis without immunohistochemistry. It is felt by Fletcher [4] that KIT immunostains should be performed as a confirmatory (if not diagnostic) measure to facilitate meaningful standardization and determination of eligibility for STI-571 therapy. KIT immunopositivity should always be interpreted in light of morphological findings. 
Careful morphological examination and clinicopathological correlation remain essential for excluding mimics and for assessment of likely behaviour of this heterogenous group of neoplasms. In the present study careful histopathological examination was done and categorized these neoplasms based on the "risk of metastasis" using the criteria described by Fletcher [4].

\section{MATERIALS \& METHODS}

The study was done for three years and we received 30 specimens consisted of resections and biopsies from gastrointestinal tract in the department of pathology and these specimens were fixed overnight in $10 \%$ formalin. Adequate sections were given from the tumour including the overlying mucosa depending on the size and location of the tumour. Both the proximal and distal resected margins were submitted for clearance. Lymphadenopathy, if any was sampled. The sampled tissue was routinely processed and paraffin embedded. Haemotoxylin and eosin stained 5micron thick sections were studied under the light microscopy and diagnosed as Gastrointestinal Stromal Tumors (GIST), for which we assessed the risk of metastasis. The proposed approach for defining risk of aggressive behaviour in GIST [4].

\begin{tabular}{|l|c|c|}
\hline \multicolumn{1}{|c|}{ Risk category } & Size & Mitotic count \\
\hline Very low risk & $<2 \mathrm{~cm}$ & $<5 / 50 \mathrm{HPF}$ \\
\hline Low risk & $2-5 \mathrm{~cm}$ & $<5 / 50 \mathrm{HPF}$ \\
\hline Intermediate risk & $<5 \mathrm{~cm}$ & $6-10 / 50 \mathrm{HPF}$ \\
& $5-10 \mathrm{~cm}$ & $<5-10 \mathrm{~cm}$ \\
\hline High risk & $>5 \mathrm{~cm}$ & $>5 / 50 \mathrm{HPF}$ \\
& $>10 \mathrm{~cm}$ & $\begin{array}{c}\text { any Mitoses } \\
\end{array}$ \\
& Any size & $>10 / 50 \mathrm{HPF}$ \\
\hline
\end{tabular}

The mitotic count is the most commonly used method to assess the proliferative activity of a tumour. Mitotic activity correlates with prognosis in GISTs. A random procedure of mitotic counting was followed.

\section{RESULTS}

The present study consisted of 30 cases of GISTs. The age of the patients ranged from 28 to 87 years with a mean of 54.6 years. Majority of cases were located in the age group of 41-50 years (30\%). Most of the patients $(80 \%)$ were more than 40 years of age.
Metastatic disease was present in six cases, among which two were associated with recurrent tumour. Among the other four cases, one was a retroperitoneal tumour with metastasis in the pubic bone, one was an ileoceacal tumour with multiple metastatic peritoneal nodules, and another was a jejunal tumour with metastatic peritoneal nodules. Yet another case of gastric GIST diagnosed on biopsy had evidence of metastasis in the lung, liver and bone on radiological examination.

Table-1: Showing the Categorisation of GISTs based on risk of metastasis

\begin{tabular}{|c|c|c|c|}
\hline Risk Grade & Criteria & No. of Cases & $\%$ \\
\hline High Risk & $\begin{array}{c}\text { Tumour size }>5 \mathrm{~cm}, \text { Mitoses }>5 / 50 \mathrm{HPF} \\
\text { Tumour size }>10 \mathrm{~cm}, \text { any Mitoses } \\
\text { Any size }>10 \text { Mitoses } / 50 \mathrm{HPF}\end{array}$ & 21 & 70 \\
\hline $\begin{array}{c}\text { Intermediate } \\
\text { Risk }\end{array}$ & $\begin{array}{c}\text { Tumour size }<5 \mathrm{~cm} \text {, Mitoses 6-10/50 HPF } \\
\text { Tumour size 5-10cm, Mitoses }<5 / 50 \mathrm{HPF}\end{array}$ & 5 & 16.67 \\
\hline Low Risk & $\begin{array}{c}\text { Tumour size } 2-5 \mathrm{~cm} \\
\text { Mitoses }<5 / 50 \mathrm{HPF}\end{array}$ & 2 & 6.67 \\
\hline $\begin{array}{c}\text { Very low risk } \\
\text { (Leiomyoma) }\end{array}$ & $\begin{array}{c}\text { Tumour size }<2 \mathrm{~cm} \\
\text { Mitoses }<5 / 50 \mathrm{HPF}\end{array}$ & & \\
\hline
\end{tabular}

There was a preponderance of high risk GISTs with 21 cases $(70 \%)$, followed by intermediate risk five cases $(16.67 \%)$, low risk two cases $(6.67 \%)$ and very low risk two cases $(6.67 \%)$.

\section{DISCUSSION}

The tumours of the GI tract which are now designated as GIST based on c-KIT and more recently on PDGFR $\propto$ mutations were described in most of the published series in the past as "Smooth Muscle Neoplasms". Only a few recent articles are titled as "GIST". This study is mainly based on morphology.
Risk categorisation as high risk, intermediate risk, low risk and very low risk is based on the size and mitotic activity [4]. The corresponding categories in the older literature are leiomyosarcoma (high risk), indeterminate or smooth muscle tumour of uncertain malignant potential (intermediate and probably low risk) and the category of very low risk probably represents those tumours which were diagnosed as leiomyomas but metastasized.

In the review of literature the study of 56 cases of smooth muscle tumours of the GI tract found that 
majority of the patients (80\%) were above the age of 40 years with maximum number of cases in the age group of 50 to 59 years [5]. Similarly, in the present study $80 \%$ of the patients were more than 40 years, but most were located in a younger age group (41 to 50 years). The age group ranged from 26 to 88 years with a mean of 64 years in a study of 51 cases by Diamond, Parks and Danton [6]. Similarly, in the present study the age ranged from 28 to 87 years but, the mean age was a decade younger (54.6 years). In few other studies of 33 cases found the age ranging from 25 to 75 years with most cases occurring in the fourth decade comparable to the present study [7]. The mean age was 58 years in a study of 200 cases of GIST [8] by somewhat comparable to the present study.

Recurrence of the disease after resection was predominantly intra abdominal and involved the original tumour site, peritoneum and liver in the study by Dematteo, Lewis and Leung et al., [9]. A similar observation was made in the present study.

In another study it has recorded three cases each of low grade and high grade leiomyosarcomas that were lessthan five $\mathrm{cms}$ in size [5]. Similarly, in our study three among the 21 high risk tumours were lessthan five cms in size. Two cases of leiomyomas in the series by Evans [5] were lessthan two cms in size one of which was oesophageal and other of rectal origin. In our study both the tumours which were lessthan two cms size were located in the oesophagus and were categorised as very low risk tumour. In one of the study it was found that duodenal leiomyosarcoma in particular were small measuring lessthan five $\mathrm{cms}$ in size [10]. But, in our series two gastric and one rectal high risk GISTs were lessthan five $\mathrm{cms}$ in size.

In the present study mitotic rate (MR) was expressed per 50 high power fields (HPFs) after counting 200 HPFs. It is difficult to make a meaningful comparision between the MR expressed in the present study and those in the past. Most of the earlier studies have expressed MR/10HPFs as originally described by Ranchod and Kempson [10]. They advocated examination of five sets of $10 \mathrm{HPFs}$ and the set with highest mitotic count was used as the final rate. This method allows for regional variation in MR that is typical of GIST. Haque and Dean [11] in their study stressed the regional variability of MR in GIST and they chose to count $200 \mathrm{HPFs}$ and express the final rate as the number of mitoses/50 HPFs. They further discuss that a mitotic rate of 5/50 HPFs appears much lower than $5 / 10 \mathrm{HPF}$ but a rate of $5 / 50 \mathrm{HPF}$ may not be equivalent to 1/10HPF. Counting mitoses in 10HPF of a mitotically active area would probably yield a higher rate than counting 200 fields, because in many GISTs the additional 190 fields would contain no mitoses at all. Regional variability of MR was observed in the present study. Franquemont in his review article has concluded that tumour categorized as intermediate risk on the basis of size and MR may be more accurately classified as high or low risk using proliferating cell nuclear antigen (PCNA) study [12].

Size and mitoses were the two criteria used in this study to classify the tumours into risk categories. Miettinen in their review article on evaluation of malignancy and prognosis of GIST have concluded that the clinical behaviour of a GIST can be predicted with relative accuracy based on the combination of tumour size and mitotic activity [13]. Both the criteria should be applied together although some small tumours, lessthan five $\mathrm{cms}$ in size with mitotic activity $<5 / 50 H P F s$ do metastasize. Significance of size is site dependent, specifically gastric tumours tend to be less aggressive than intestinal tumours, even those lessthan five cms in size, provided that their mitotic activity is no more than 5/50 HPFs. They have also stated that GISTs lessthan two cms have negligible mitotic activity (usually <5 / 50 HPF). Such tumours are largely benign at all sites when completely removed. In 1985 one study has stated that malignant gastrointestinal smooth muscle tumours had diameters as small as one $\mathrm{cm}$. and maximal mitotic rates as low as one mitotic figure per ten high power fields, thus suggesting that these parameters cannot be relied upon in the differential diagnosis [5]. Ranchod and Kempson were unable to arrive at definitive criteria for malignancy [10] and other authors who did separate leiomyomas from leiomyosarcomas, accepted occasional metastasizing neoplasms in their 'benign' category [14]. In the present study it was observed that high risk small intestinal GISTs (five cases) exhibited lessthan five mitoses / 50HPFs. Also two among six tumours with metastasis had fewer mitoses ( $<5 / 50$ HPFs) and three among the 21 high risk cases were lessthan five $\mathrm{cms}$ in size.

\section{CONCLUSION}

To conclude the tumours were classified into "risk of metastasis categories" based on the size of the tumour and mitotic activity. Identifying mitotic figures and counting them was associated with problems and were due to uneven distribution of the mitotic figures, variable thickness of the section and presence of inflammatory cells. Inspite of the difficulty mitoses is still considered as a good prognostic marker. The other features which were useful in risk categorisation were cellularity, nuclear atypia, tumour cell necrosis and mucosal invasion. Since this study did not include follow-up, the significance and outcome of such a risk categorisation could not be determined.

\section{REFERENCES}

1. Stout, A. P. (1962). Bizarre smooth muscle tumors of the stomach. Cancer, 15(2), 400-409.

2. Mazur, M. T., \& Clark, H. B. (1983). Gastric stromal tumors. Reappraisal of histogenesis. The American journal of surgical pathology, 7(6), $507-$ 519. 
3. Herrera, G. A., De Moraes, H. P., Grizzle, W. E., \& Han, S. G. (1984). Malignant small bowel neoplasm of enteric plexus derivation (plexosarcoma). Digestive diseases and sciences, 29(3), 275-284.

4. Fletcher, C. D., Berman, J. J., Corless, C., Gorstein, F., Lasota, J., Longley, B. J., ... \& Shmookler, B. (2002). Diagnosis of gastrointestinal stromal tumours: a consensus approach. Hum pathol, 33(5), 459-465.

5. Evans, H. L. (1985). Smooth muscle tumors of the gastrointestinal tract. A study of 56 cases followed for a minimum of 10 years. Cancer, 56(9), 22422250.

6. Diamond, T., Danton, M. H., \& Parks, T. G. (1990). Smooth muscle tumours of the alimentary tract. Annals of the Royal College of Surgeons of England, 72(5), 316-320.

7. Shah, P., Shah, A., \& Wani, N. A. (1995). Smooth Muscle Tumours of the Gastrointestinal Tract (A Clinico Pathological Study of 33 Cases). Indian J Surg, 95-99.

8. Demetri, G. D., Von Mehren, M., Blanke, C. D., Van den Abbeele, A. D., Eisenberg, B., Roberts, P. J., ... \& Fletcher, J. A. (2002). Efficacy and safety of imatinib mesylate in advanced gastrointestinal stromal tumors. New England Journal of Medicine, 347(7), 472-480.
9. DeMatteo, R. P., Lewis, J. J., Leung, D., Mudan, S. S., Woodruff, J. M., \& Brennan, M. F. (2000). Two hundred gastrointestinal stromal tumors: recurrence patterns and prognostic factors for survival. Annals of surgery, 231(1), 51-58.

10. Ranchod, M., \& Kempson, R. L. (1977). Smooth muscle tumors of the gastrointestinal tract and retroperitoneum. A pathologic analysis of 100 cases. Cancer, 39(1), 255-262.

11. Haque, S., \& Dean, P. J. (1992). Stromal neoplasms of the rectum and anal canal. Human pathology, 23(7), 762-767.

12. Franquemont, D. W. (1995). Differentiation and risk assessment of gastrointestinal stromal tumors. American Journal of clinical pathology, 103(1), 41-47.

13. Miettinen, M., Sobin, L. H., \& Lasota, J. (2002). Evaluation of malignancy and prognosis of gastrointestinal stromal tumors: a review. Human pathology, 33(5), 478-483.

14. Amin, M. B., Ma, C. K., Linden, M. D., Kubus, J. J., \& Zarbo, R. J. (1993). Prognostic Value of Proliferating Cell Nuclear Antigen Indexin Gastric Stromal Tumors: Correlation With Mitotic Count and Clinical Outcome. American journal of clinical pathology, 100(4), 428-432. 